\title{
DÜBLIN
}

Technological University Dublin

ARROW@TU Dublin

Articles

School of Electrical and Electronic Engineering

2019

\section{Towards an ontology for soft robots: What is soft?}

\author{
Kevin Chubb \\ Technological University Dublin, kevin.chubb@tudublin.ie \\ Damon Berry \\ Technological University Dublin, damon.berry@tudublin.ie \\ Ted Burke \\ Dublin Institute of Technology, ted.burke@tudubln.ie
}

Follow this and additional works at: https://arrow.tudublin.ie/engscheleart2

Part of the Electrical and Computer Engineering Commons

\section{Recommended Citation}

Chubb K, Berry D, Burke T. Towards an ontology for soft robots: what is soft? Bioinspir Biomim. 2019 Oct 25;14(6):063001. doi: 10.1088/1748-3190/ab483f. PMID: 31557734.

This Article is brought to you for free and open access by the School of Electrical and Electronic Engineering at ARROW@TU Dublin. It has been accepted for inclusion in Articles by an authorized administrator of ARROW@TU

Dublin. For more information, please contact

arrow.admin@tudublin.ie, aisling.coyne@tudublin.ie, gerard.connolly@tudublin.ie.

Funder: Technological University Dublin

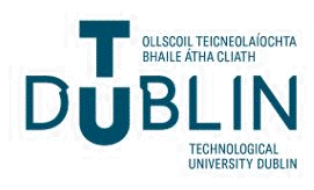


PERSPECTIVE

\section{Towards an ontology for soft robots: what is soft?}

To cite this article: Kevin Chubb et al 2019 Bioinspir. Biomim. 14063001

View the article online for updates and enhancements.
You may also like

$$
\begin{aligned}
& \text { - Soft and smart modular structures } \\
& \frac{\text { actuated by shape memory alloy (SMA) }}{\text { wires as tentacles of soft robots }} \\
& \text { Hu Jin, Erbao Dong, Min Xu et al. } \\
& \text { - Soft robot with a novel variable friction } \\
& \frac{\text { design actuated by SMA and }}{\text { electromagnet }} \\
& \text { Junfeng Li, Wenkai Chang and Qiyu Li }
\end{aligned}
$$

Scalable fabric tactile sensor arrays for soft bodies

Nathan Day, Jimmy Penaloza, Veronica J

Santos et al.

\section{Recent citations}

- Seongqun Joe et al

- Soft Robotic Manipulators: Designs. Actuation, Stiffness Tuning, and Sensing Weiqiang Dou et al

A Magnetorheological Fluid-Filled Soft Crawling Robot With Magnetic Actuation Dezheng Hua et al

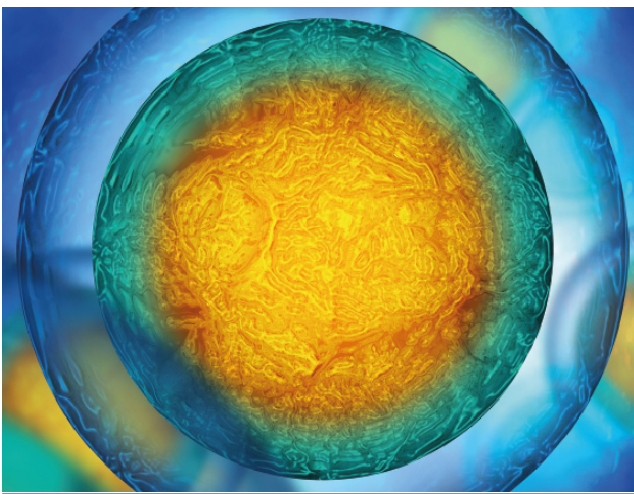

Biophysical Society IOP $\mid$ ebookS

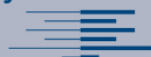

Your publishing choice in all areas of biophysics

research.

Start exploring the collection-download the first chapter of every title for free.

This content was downloaded from IP address 80.233 .40 .131 on $01 / 12 / 2021$ at 08:55 


\title{
Bioinspiration \& Biomimetics
}

\section{PERSPECTIVE}

\section{Towards an ontology for soft robots: what is soft?}

\section{May 2019 \\ REVISED \\ 7 August 2019 \\ ACCEPTED FOR PUBLICATION \\ 26 September 2019 \\ PUBLISHED}

25 October 2019

\author{
Kevin Chubb ${ }^{1,2} \odot$, Damon Berry ${ }^{1} \oplus$ and Ted Burke ${ }^{1} \odot$ \\ 1 School of Electrical and Electronic Engineering, Technological University Dublin, Kevin Street, Dublin 8, Ireland \\ 2 Address correspondence to: Greenway Hub, TU Dublin, Grangegorman, Dublin 8, Ireland \\ E-mail:kevin.chubb@tudublin.ie
}

Keywords: soft robotics, bioinspired robot, smart material

\begin{abstract}
The advent of soft robotics represents a profound change in the forms robots will take in the future. However, this revolutionary change has already yielded such a diverse collection of robots that attempts at defining this group do not reflect many existing 'soft' robots. This paper aims to address this issue by scrutinising a number of descriptions of soft robots arising from a literature review with the intention of determining a coherent meaning for soft. We also present a classification of existing soft robots to initiate the development of a soft robotic ontology. Finally, discrepancies in prescribed ranges of Young's modulus, a frequently used criterion for the selection of soft materials, are explained and discussed. A detailed visual comparison of these ranges and supporting data is also presented.
\end{abstract}

\section{Introduction}

Soft robotics is an emerging field which promises to create robots that are radically different from conventional robots found today. Where conventional robots comprise rigid materials and often perform tasks in controlled environments while isolated from humans, soft robots may operate in close proximity to humans due to the inherent safety provided by their ability to readily deform. The ability of soft robots to deform (by design) in response to external forces may enable some robots to finally become common in domestic settings, may enable greater search and rescue devices, may help create better wearable devices and prostheses, or may improve the utility of manipulators in confined spaces.

Described as 'open and free of dogmatic restrictions to any constrained set of methods, principles, or application domains' [1], the freedom provided by this new field of robotics is reflected in the diversity of robots built by researchers and hobbyists. However, this freedom presents challenges. Numerous attempts to define this diverse family of robots has resulted in a labyrinth of descriptions in the literature. Despite a single term distinguishing this branch of robotics from others, soft is difficult to define as it has many possible interpretations. Is soft only used as an intuitive term to separate this class of robots from conventional robots? Is softness related to material compliance, structural compliance or both? In each of these cases, how does hardness or soft matter fit in? The authors aim to resolve this ambiguity by examining a selection of concepts emerging from descriptions of soft robotics in the literature and clarifying exactly what is meant by soft.

A clear description of soft may clarify the boundary between soft robotics and conventional robotics. However, within this burgeoning field, a large number of soft robots have been built in a variety of unconventional and seemingly unrelated forms for a range of different applications. This paper also aims to initiate the categorisation of existing soft robotics so that this field may become more structured. This categorisation is not intended to restrict the opportunity for creativity found in soft robotics but rather, to provide a structure so that an understanding of this complex field becomes more accessible.

Finally, in the course of the following discussion, a common description of existing soft robots is introduced: compliance. Young's modulus, a quantitative measure of this property, is scrutinised. Ranges of values for this property have been used to select materials safe for physical interaction with humans [1] and in a definition of soft robots [2]. However, proposed ranges of values for this criterion differ by orders of magnitude. The final objective of this paper is to clarify how this discrepancy may have arisen. Additionally, the suitability of this criterion to some classes of soft robots is also discussed.

The purpose of these three objectives is to strive towards a unified understanding of the breadth and 
complexity of soft robotics for researchers active in this domain. This paper aims to begin this process by defining the boundary of soft robotics through an investigation into the term soft, identifying a relationship between existing soft robots, and clarifying the use of Young's modulus for robots in this field.

\section{What is soft?}

A good starting point is to begin with the physical forms soft robots have taken rather than with their descriptions, especially if these descriptions are to be under scrutiny. Figure 1 shows a selection of robots described by their creators as 'soft' in the literature, grouped into tiers according to their similarities. Small differences separate neighboring devices in figure 1 but the differences between devices at the extremities of this spectrum are substantial. It could be argued that some devices in this figure, especially those in Tier 1 , do not belong in a discussion about soft robotics. Many of the descriptions provided in the following section exclude these types of robot because their compliance is achieved not through the use of soft materials but by pairing active $(\mathbf{H})$ and passive (I) structural compliance with rigid materials. However, both of these approaches are considered by some to be soft $[10,12-14]$. Figure 1 is intended as a tool to display the variety of 'soft' robots in the literature but it is by no means exhaustive. Row-bot [15], for example, is not included. This robot employs soft materials in its microbial fuel cell, in its fuel intake 'mouth', and for locomotion. However, it is not identified as a 'soft' robot by its creators. Row-bot is a biomimetic autonomous rigid robot that requires soft materials to function. Figure 1 is intended as an aid to understand the breadth of robots that are distinguished as 'soft' in the literature; this and similar robots have not been included.

\section{Compliance}

Despite their differences, all of the robots in figure 1 share a common trait: compliance. This term has been used frequently in the literature to describe soft robots and their supporting technologies [1, 2, 4, 7, 16-26]. Soft robots have sometimes been described exclusively in terms of the compliance of their comprising materials $[1,2,4,17,21,24,26]$ while others attribute a robot's softness to both structural and material compliance $[16,17,24]$. In both cases, compliance is often referred to without explicitly describing its meaning. For the purpose of determining a clear meaning for soft, compliance will be discussed briefly with the aim of determining whether material and/or structural compliance is applicable to soft robots.

Stiffness, the inverse of compliance [27], represents the resistance of an object to non-permanent (elastic) deformation when under stress. As an extensive property, i.e. one that depends upon the amount of material present, the elastic modulus of a material and its geometry affect a material's stiffness. Geometry describes the dimensions of the material and a material's elastic modulus is an intensive characteristic of the material; representing the relationship between an applied stress and the resultant elastic deformation of a test specimen of standardized geometry. Figure 2 shows two solid bars of different cross-section fixed to an anchor. Assuming that both bars are made from the same material, one may surmise the effect of geometry on a body's stiffness. Formally, in this specific case deflection $\delta$ due to force $F$ is given by [28]:

$$
\delta=4 F \frac{1}{E} \frac{l^{3}}{b h^{3}}
$$

where $E$ is the Young's modulus of the material and $\frac{l^{3}}{b h^{3}}$ relates to the geometry of each of the two bars as annotated by figure 2. In a structure, compliance depends upon the stiffness of each member element as well as the location and arrangement of these elements in the structure. An example of this is a tower crane, where both the stiffness of the individual steel bars and their arrangement into a truss affect the crane's stiffness.

If a robot is considered soft based on a measure of its materials' compliance alone-using the Young's modulus of its constituent materials for example [2]one should consider how appropriate this description is to soft robots. Material compliance describes only how easily a material elastically deforms under stress. Soft robots typically comprise a number of materials of differing compliance arranged into a structure. The compliance of the assembled robot not only depends upon the elastic modulus, but the geometry and structure of the device; both material and structural compliance should be considered. However, a robot's compliance can be more complex: the deformation of an assembled robot under stress may depend upon its orientation to that stress i.e. it may be anisotropic. This may arise due to asymmetrical geometry, the orientation of embedded materials like glass fibres, grains in materials like woods or metals, or the specific arrangement of elements forming a structure. Soft robots may comprise parts that differ in composition and geometry, each requiring a matrix of terms to describe the local compliance in each direction. Furthermore, many devices in figure 1 could be considered compliant in some directions while stiff in others. Although considered soft robots, these robots may not act as a soft robot in all directions. A detailed description of how a soft robot achieves compliance and in which direction it is compliant is required to determine when it will interact softly with its target and when it will not. Additionally, soft robots are never passive devices. Soft robots such as $(\mathbf{A})-(\mathbf{C})$ and $(\mathbf{E})-(\mathbf{G})$ in figure 1 employ fluid-chamber actuators. When the pressure in a chamber changes, the compliance of the robot also changes. Soft robots can also modify their stiffness by employing materials that undergo phase changes $[29,30]$, using particle jamming [6], increasing ten- 
Tier 4 - Entirely soft robots, independent of any hard elements.

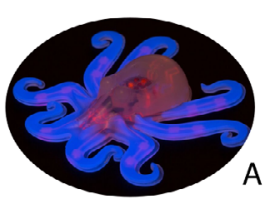

Tier 3 - Entirely soft robots relying on external hard components.

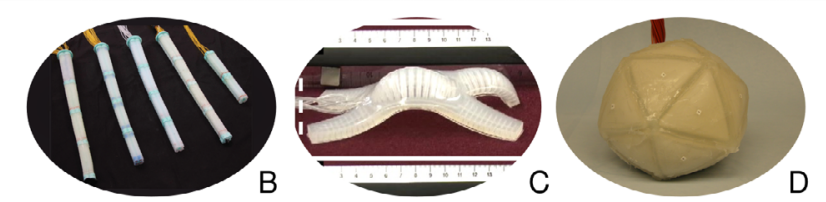

Tier 2 - Robots with primary functions fully or partially achieved through soft elements making up a significant portion of their body.
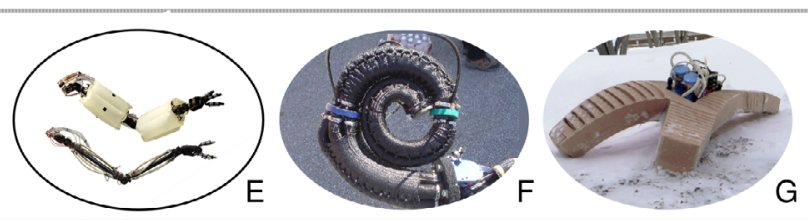

Tier 1 - Conventional robots modified to achieve compliance actively through controlled joint impedance or passively through compliant structural elements.

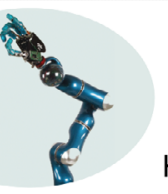

$\mathrm{H}$

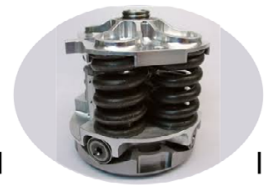

Figure 1. Octobot (A) is an untethered robot comprising only soft components [3]. Marchese and Rus's soft spatial manipulator (B), the multigait soft robot $(\mathbf{C})$ (a precursor to $(\mathbf{G})$ ), and the spherical jamming mobile robot (D) comprise only soft materials but require external supplies [4-6]. The lightweight soft robotic arm (E) moves using pneumatic actuators that also provide a compliant covering [7]. OctArm (F) uses pneumatic actuators to create a continuum manipulator but requires rigid plates to separate each section of the manipulator [8]. The resilient untethered soft $\operatorname{robot}(\mathbf{G})$ moves using pneumatically powered actuators but relies on hard batteries, compressors, and micro-controllers [9]. DLR Robotics' LWR-III (H) [10] achieves softness through lightweight materials, sensor redundancy, and active compliance control. Variable impedance actuators (I) incorporate compliance into a rigid robot, creating a * soft' robot, by exploiting passive or actively controlled elastic elements [11]. (Credits: (A) Lori K Sanders, Michael Wehner, and Ryan L Truby, Harvard University; (B) reproduced from [4] with permission, Copyright 2016, SAGE Publications; (C) Robert F Shepherd; (D) used with permission by the authors; (E) Yong-Lae Park, Carnegie Mellon University; (F) reproduced from [8] with permission, Copyright 2006, IEEE; (G) reproduced from [9] with permission, Copyright 2014, Mary Ann Liebert, Inc.; (H) reproduced from [10] with permission, Copyright 2008, IEEE; (I) reproduced from [11] with permission, Copyright 2009, IEEE.)

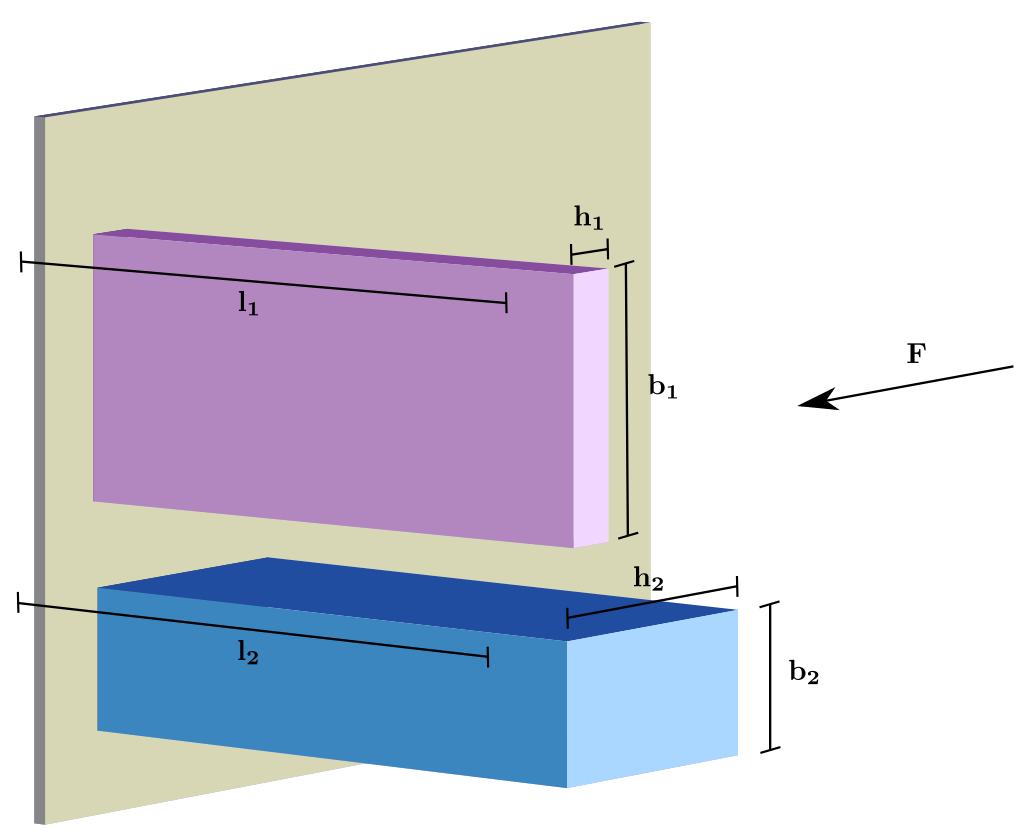

Figure 2. Stiffness of cantilever beams.

sion on tendons [31], or controlling the impedance of joints [10].

If a soft robot is compliant, one should consider how specifically it achieves compliance. The softness of these devices should not be vaguely attrib- uted to material or structural compliance and defined by incomplete criteria for these characteristics. In a type of robotics with safe physical human interaction in the crosshairs, an incomplete understanding of a soft robot's compliance may result in unsuitable 
interaction with humans. As soft robotics creates interest in people from many varied fields, the literature describing soft robots should become accurate and complete when describing compliance. Literature enabling readers to fully understand compliance may nurture the creation of more diverse forms of soft robots. Conversely, literature describing the compliance of soft robots without a comprehensive description of this term may create unnecessary confusion and so hinder the field's development. Soft robots cannot be accurately described according to the compliance of materials alone; this is a simplification. Many existing soft robots comprise materials assembled into structures that change in compliance during actuation.

Soft robotics provides ample opportunity for the creation of radically new forms of robots for many varied applications. Highlighting the complex mechanical properties of soft robots may reveal new opportunities to gain further control over a soft robot's deformation. When first considering these mechanical characteristics, it is possible to perceive rigidity as an undesirable property in soft robots. However, in our view, it is beneficial to view rigidity as an adjustable property which may enable a soft robot to deform appropriately. Similar to (I) in figure 1 [11], soft robots may be constructed, with what may be considered quite stiff materials, if a compliant structure enables a robot to deform. Structures may be useful to modify the compliance of a soft robot but also to reorient the direction in which a robot may deform under stress. This anisotropic deformation may be useful to direct and control how a robot deforms. For example, by introducing organised structures and/or stiff materials into a soft material, deformation may be directed to create actuators [32], control the locomotion of mobile robots [9] or may reduce the computational load required to determine a soft robot's kinematics [33]. Origami and kirigami, the art of folding paper and the art of cutting paper, respectively, have been identified as means of simplifying the fabrication of compliant and reconfigurable structures [34]. Described as having the properties of both soft and hard robots, origami and kirigami robots have been described as a distinct class of robot which self-assemble from a flat sheet [34]. However, origami, kirigami, and mechanical metamaterials may be used to introduce passive compliant structures into soft robots; to enable relatively complex locomotion from simple actuation [35-37] or to modify the bulk mechanical properties of materials [38-40] for example. Additionally, rigid components may be used in tandem with soft materials to create soft robots which benefit from the advantages of both materials [7]. Also drawing inspiration from the art world, tensegrity structures consist of a combination of cables under tension and separated rigid elements under compression. These structures have been identified as having a number of properties which are useful for cobots and soft robots [41] and have been used to fabricate a number of mobile soft robots [42-44].

\section{Softness}

An obvious question at this point in the discussion is: if soft robots are compliant, where does softness fit in? In order to answer this question one should consider the origin and development of soft robots as well as the potential meanings of soft. The words 'soft robot' were originally used to describe a rigid hand device employing McKibben pneumatic actuators [45]. 'Soft robotics' was later used to describe the active structural compliance programmed into rigid robots such as LWR-III [46]. Many current 'soft' robots employ compliant materials and structures to achieve softness. A branch of soft robotics known as soft matter robotics [47] (also named soft material robotics [48] despite a distinction between matter and material being made by Kastor et al [49]) exclusively employs a specific class of matter to become 'soft' [47]. The meaning of 'soft' changes between each of these contexts. Its original use in [45] may have been as an intuitive term to distinguish this device from conventional robots. Intuitively, one may describe something soft in a range of ways: pleasing to touch, gentle, and easily moulded or deformed. Currently, compliance is the prevalent characteristic distinguishing soft robots from conventional robots. Just as compliance is the inverse of stiffness, softness can be interpreted as the inverse of hardness; a property describing the resistance of a material to local permanent (plastic) deformation when under stress. In the literature relating to soft robotics, the property of hardness has not, to the best of the authors' knowledge, been used to define robots in that class. Rather, plastic deformation has been used to describe phase change materials, such as shape memory alloys [50], contained fluids in soft fingers [51], and when using of hot melt adhesives and contained granular media in grippers [52]. In one definition of soft materials, both the elastic and plastic deformation of a material are considered [49]. However, save for shore hardness when selecting elastomers (e.g. [53]), hardness does not feature in the description of soft robots. Soft matter robots are 'soft' because of their relatively large non-linear response to relatively small forces [54, pp 1-7]. Although initially seeming inaccessible, examples of soft matter include foams, milk, elastomers, and ferrofluids; belonging to foams, colloids, polymers, and surfactants, respectively. Each of these descriptions of 'soft' may be valid for a number of robots but not one can cover all of the example 'soft' robots provided in figure 1. Due to the immaturity of soft robotics, descriptions of 'soft' change readily. The meaning of 'soft' not only changes with the advances in this field but also changes with the researcher's area of interest. As Wang and Iida aptly put it, most reviews of soft robotics collected ' case studies that fell into ambiguous classifications of softness based on the authors' individual experiences and research topics' [47].

In order to answer the title question, and in so doing encourage debate on this topic, one may look 


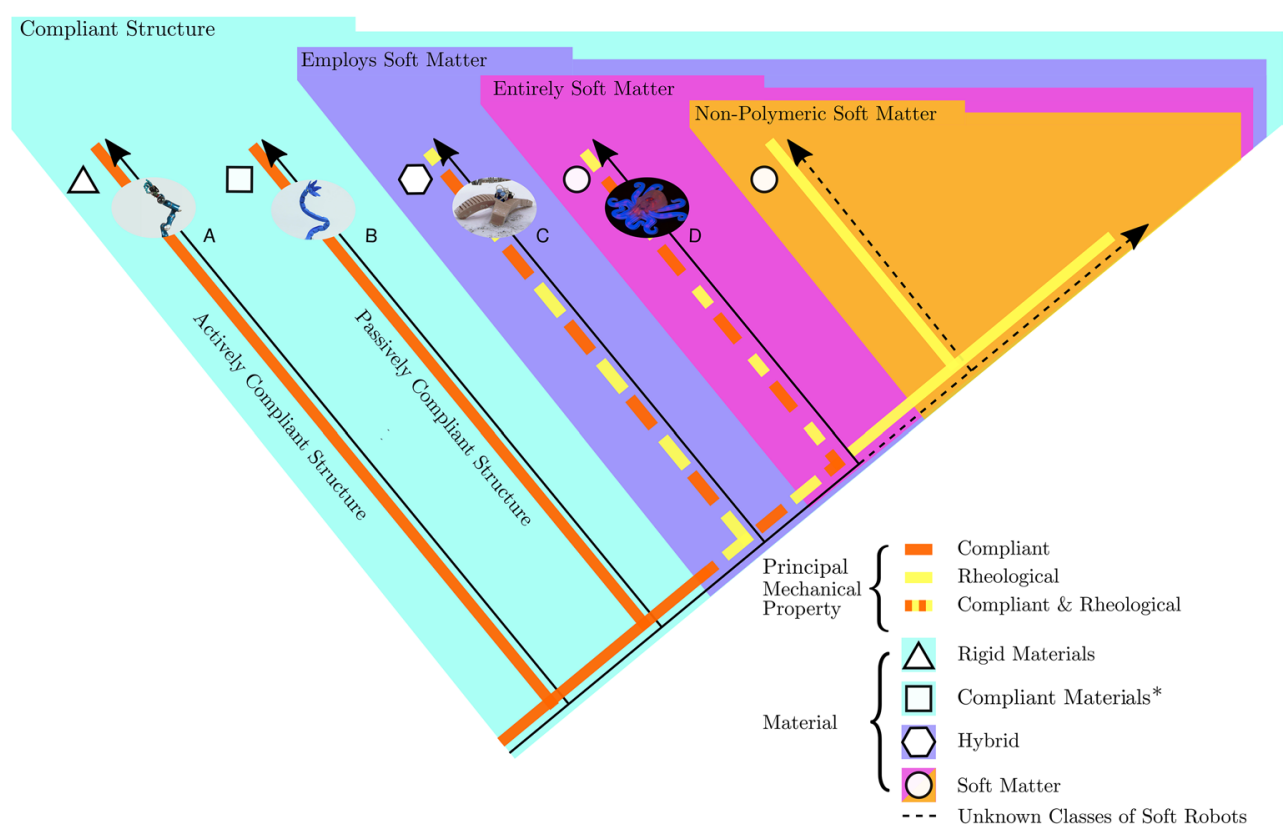

Figure 3. A categorisation of existing soft robots. Each branch in this diagram represents a distinct approach towards achieving the characteristic intentional deformation of soft robots. Each colored section represents the principal characteristic of the contained branches, where branches in each successive section may possess the traits of the preceding sections. Examples in each branch are: DLR Robotics' LWR-III (A) [10], fluid driven origami-inspired artificial muscles (B) [32], resilient untethered soft robot (C) [9], and Octobot (D) [3].*Materials of varying compliance may be used in the construction of passively compliant structures. However, it is possible to create a passively compliant structure without relying on material elasticity. If the magnetic field of a permanent magnet restores a structure to its original configuration, the deformation of this structure may resemble that of a passively compliant structure. (Credits: (A) reproduced from [10] with permission, Copyright 2008, IEEE; (B) Wyss Institute at Harvard University; (C) reproduced from [9] with permission, Copyright 2014, Mary Ann Liebert, Inc.; (D) Lori K. Sanders, Michael Wehner, and Ryan L. Truby, Harvard University.)

towards possible future developments in soft robots. The authors choose the potential provided by soft matter for this purpose. Currently soft matter robots frequently comprise polymers while robots comprising colloids or smart fluids are largely undeveloped [47]. Soft robots in the near future may further explore and aim to control these relatively unused materials. In soft matter, the rheological properties of the matter are of concern [54, p 165]; where rheology refers to the flow and deformation of matter under stress [55]. Soft matter describes materials exhibiting soft mechanical properties on a macroscopic scale [56]. Future soft robots constructed from swarms of hard, stiff robots at the mesoscopic scale, $1-1000 \mathrm{~nm}$ [56], may also exhibit soft characteristics at a macroscopic scale. Majidi suggests that as soft robots are further miniaturized the complex behavior of soft matter robots will be described by the elasticity and rheology of soft matter [1].

What is soft? A descriptive answer must be given as one quantitative criterion cannot be used to define each distinct approach towards achieving softness. Soft relating to soft robots is a characteristic describing the intentional deformation of robots when under certain stress or stresses. The stress or stresses in response to which a soft robot may deform is specific to the desired application of a soft robot; for example, a soft robotic manipulator may be required to bear certain loads but deform under impacts and conform to irregularly shaped objects. Deformation may be either elastic or plastic, where many currently developed soft robots deform elastically. In this case, compliance is the defining characteristic of soft robots. Soft matter robots are considered a class of soft robots where soft matter is employed as the deforming component of a soft robot. Soft matter comprises a large diverse group of materials from viscous liquids, to gels, to viscoelastic solids. Currently, many soft matter robots use elastomers but many more materials may be exploited in the future. Softness is currently achieved in many ways, from the control of joint impedances creating an actively controlled compliant structure [10] to robots partially [9] or fully [3] comprising soft matter. With advances in the control of soft matter, or perhaps with the creation of smart soft matter using swarms of tiny robots, the rheological properties of these devices will become prominent.

Figure 3 is a diagram displaying a categorisation of existing soft robots. Each branch on this diagram represents a distinct type of soft robot. The first class, where a robot's characteristic softness is primarily achieved through the compliance of its structure, is split into two branches: active and passive structural compliance. Active structural compliance stems from the control of a robot's actuation; DLR Robotics' LWR-III [10] for example, employs this control scheme. Passive structural compliance emerges from the mechanical properties of structures such as springs 
[11], tensegrity structures [43, 44], origami [32], kirigami [57], and other mechanical metamaterials [37]. The materials used in these structures may vary in compliance from the relatively rigid spring steels and compression members used in [11] and [44], respectively, to the fabrics, plastics, and cardboard used in origami [32] and kirigami [57]. Compliant structures, active and passive, are not solely found within these classes of soft robots. Active compliance control $[29,58]$, origami [59], kirigami [36], and mechanical metamaterials [60] have been used in robots employing soft matter. A number of systems in the 'Employs Soft Matter' category, particularly mobile soft robots, rely on rigid components to function; for example $[5,6,9,30,61]$. Being a relatively new field, many supporting technologies which are soft lack the performance of their rigid counterparts [7, 62, 63]. Some existing 'entirely soft robots' (e.g. [5]) may not even be considered robots unless the tethered rigid components which grant them autonomy are included [64]. As a consequence, the development of a practically useful and entirely soft mobile robot remains a grand challenge of soft robotics. Until technologies supporting the development of soft robots-power generation, computation, and actuation mature-the capabilities, autonomy, and mobility of an entirely soft robot are likely to be limited (e.g. [3]).

Soft robots have been identified as having the potential to outperform their rigid counterparts in uncontrolled environments [65], when operating closely with humans and when mimicking biological systems [66]. When soft robotics finally provides practical platforms for these applications, the degree of autonomy in these robots may be questioned. Currently, achieving autonomy in an entirely soft mobile robot poses a challenge [63]. However, when such obstacles are overcome, soft robots of the future may have unprecedented access to humans or other natural systems. Although potentially safe, soft robots have the capability to act dangerously. How a soft robot may make appropriate decisions while total information about their state may not be observable [65] raises concerns over how predictable a soft robot's actions may be. Concerns have also been raised surrounding the degree of allowable autonomy in robots designed for physical human-robot interaction [67]. The advent of soft robotics adds a new dimension to this discussion. With such diversity in soft robotics, a soft robot's degree of softness (or potential for harm) may affect how much autonomy may be allowed in a given environment. The definition of soft robots proposed in this paper depends upon a soft robot's deformation being fit for its intended application. If all scenarios within which a soft robot may operate cannot be anticipated or if a soft robot relies upon rigid components, limitations on a soft robot's autonomy may be purposefully imposed to ensure its safe operation.

\section{Young's modulus}

Compliance is used to describe many soft robots but both compliance and its inverse, stiffness, are relative terms. In order to design a compliant soft robot for a specific application or to operate within a specific environment, these terms should have an appropriate reference frame. The aim of this section is to scrutinize a quantitative measure provided frequently in the literature for this purpose: Young's modulus [1-3, 68, 69].

Young's modulus is the constant of proportionality between the stress and strain of a linearly elastic material undergoing elastic deformation. An object's stiffness is proportional to the constituent material's Young's modulus; where the object's geometry also influences its stiffness $[27,70]$. As Young's modulus is an intensive characteristic, i.e. independent of the amount of the material present, it is a useful measure to compare linearly elastic materials' resistance to elastic deformation. Majidi used a range of Young's moduli for selecting materials suitable for soft robots that are designed for safe physical human-robot interaction [1]. Majidi suggests that soft robots designed for this purpose should match their constituent materials' Young's moduli to that of soft biological material. Majidi supports this by proposing that 'contacting materials should share similar mechanical rigidity in order to evenly distribute internal load and minimize interfacial stress concentrations'. Majidi provides a range of Young's moduli between $10^{2}-10^{6} \mathrm{~Pa}$ as this reference. In their seminal paper, Rus and Tolley follow Majidi's compliance matching principle but define all soft robots as 'systems that are capable of autonomous behavior, and that are primarily composed of materials with moduli in the range of that of soft biological materials'; providing a different range of $10^{4}-10^{9} \mathrm{~Pa}$ [2]. The limitations of Young's modulus as a defining characteristic of soft robots and soft matter are acknowledged by both Majidi [1] and Rus and Tolley [2]. However, scrutiny is still required. Firstly, these reference frames, based on the same principle, differ by orders of magnitude. McKee et al may provide an explanation for such a large difference between these ranges, hypothesizing that bulk and local characteristics of heterogeneous biological tissue are captured differently by indentation and tensile methods [71] Figure 4 displays data provided by Majidi [1], Rus and Tolley [2], and McKee et al [71]. Young's modulus is tested using homogeneous, uniform cross-section bars that are subject to axial loading and small deformations to ensure elastic deformation [1]. The mechanical properties of soft biological material differs from those of the test specimens. Skin, for example, behaves as a non-homogeneous, anisotropic, and non-linear viscoelastic material [72]. Majidi suggests matching a candidate material's mechanical rigidity with that of 


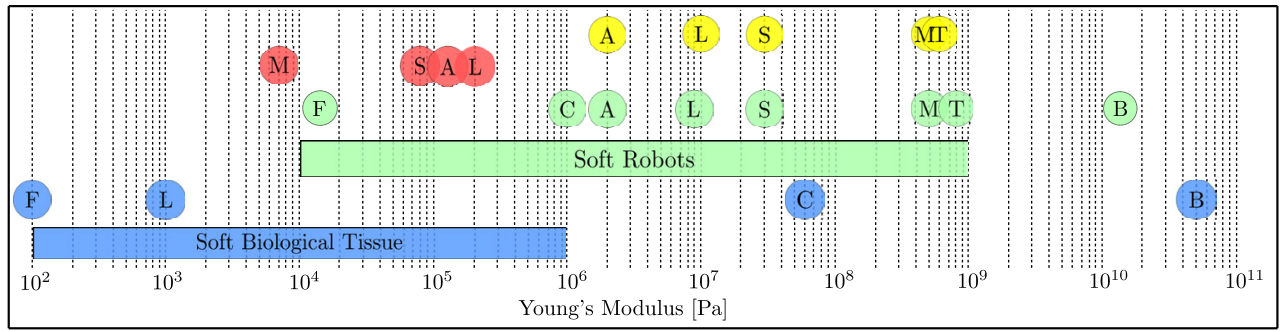

$\begin{array}{lll}\text { McKee et al. Tensile [71] } & \mathrm{F}=\text { Fat } & \mathrm{T}=\text { Tendon } \\ \text { McKee et al. Indentation }[71] & \mathrm{L}=\text { Liver Cells } & \mathrm{B}=\text { Bone } \\ \text { Rus \& Tolley }[2] & \mathrm{M}=\text { Muscle } & \mathrm{C}=\text { Cartilage } \\ \text { Majidi [1] } & \mathrm{S}=\text { Skin } & \mathrm{A}=\text { Artery }\end{array}$

Figure 4. The suggested ranges of Young's moduli by Majidi and Rus and Tolley are represented here by bars spanning these ranges. The Young's modulus of each biological sample, as provided by its respective author, is represented by a circle placed approximately at its measured value. Data provided by each author are represented using assigned color codes. Majidi's and Rus and Tolley's data are represented by blue and green while McKee's tensile and indentation data are represented by yellow and red, respectively. The data provided by McKee et al represents the average value of the samples reviewed; without outlier values. The tensile data provided by McKee et al appears to align with Rus and Tolley's data. It is not clear whether Majidi's data aligns with either the tensile or indentation data provided by McKee et al.

soft biological material in order to create a soft robot that may interact safely with a human, but rigidity alone is not sufficient when such complex behavior is exhibited by soft biological material; Young's modulus should not be used in isolation. Furthermore, defining an entire class of robots-independent of their purpose-by this criterion not only excludes many soft robots that are achieving their desired compliance through alternative means (see figure 1) but does not take into account the wide variety of contexts in which each robot operates.

It is useful at this point to align the discussion of Young's modulus to the previous discussion on compliance. Compliance is the inverse of stiffness, a property dependent on both a material's Young's modulus and its geometry. Young's modulus is a useful property to compare the reaction of these materials to an applied tensile stress or stress arising from indentation however it does not fully describe an assembled robot's stiffness. The elastic modulus and geometry of each member material, the arrangement of these members into the assembled structure, and a robot's actuation should be considered in an assembled robot. If an assembled robot comprises materials that are not linearly elastic and homogeneous, additional properties may be required. If the safe use of a robot is justified based on its compliance, then the test method should be stated.

\section{Conclusions}

Soft robotics is a developing field that is still maturing. The meaning of soft has changed as the field has advanced. In many existing soft robots, compliance is the definitive characteristic. However for a soft robot, compliance cannot be attributed to intensive material characteristics alone: material, structural, passive and active characteristics should be considered. With greater inclusion of soft matter in soft robotics, the rheological properties of soft robots will become prominent. A categorisation of soft robots according to their principal mechanical property, material composition, and their principal means of achieving softness is presented in this paper. For soft robots based on material compliance, the authors encourage precision in the use of Young's modulus. The authors suggest that the method of testing Young's modulus should be explicit and justified when selecting compliant materials for safe human-robot physical interaction.

\section{Acknowledgments}

This work was funded by the TU Dublin College of Engineering \& Built Environment Postgraduate Scholarship Scheme and by the School of Electrical \& Electronic Engineering.

\section{Author disclosure statement}

No competing financial interests exist.

\section{ORCID iDs}

Kevin Chubb (1) https://orcid.org/0000-0002-62121065

Damon Berry (1) https://orcid.org/0000-0002-2290-7661

Ted Burke (D) https://orcid.org/0000-0002-4806-3436

\section{References}

[1] Majidi C 2014 Soft robotics: a perspective-current trends and prospects for the future Soft Robot. 15-11

[2] Rus D and Tolley M T 2015 Design, fabrication and control of soft robots Nature 521467

[3] Wehner M, Truby R L, Fitzgerald D J, Mosadegh B, Whitesides G M, Lewis J A and Wood R J 2016 An integrated design and fabrication strategy for entirely soft, autonomous robots Nature 536451 
[4] Marchese A D and Rus D 2016 Design, kinematics, and control of a soft spatial fluidic elastomer manipulator Int. J. Robot. Res. 35 840-69

[5] Shepherd R F, Ilievski F, Choi W, Morin S A, Stokes A A, Mazzeo A D, Chen X, Wang M and Whitesides G M 2011 Multigait soft robot Proc. Natl Acad. Sci. 108 20400-3

[6] Steltz E, Mozeika A, Rodenberg N, Brown E and Jaeger H M 2009 Jsel: jamming skin enabled locomotion IEEE/RSJ Int. Conf. on Intelligent Robots and Systems (IEEE) pp 5672-7

[7] Ohta P, Valle L, King J, Low K, Yi J, Atkeson C G and Park Y-L 2018 Design of a lightweight soft robotic arm using pneumatic artificial muscles and inflatable sleeves Soft Robot. 5 204-15

[8] McMahan W, Chitrakaran V, Csencsits M, Dawson D, Walker I D, Jones B A, Pritts M, Dienno D, Grissom M and Rahn C D 2006 Field trials and testing of the OctArm continuum manipulator IEEE Int. Conf. on Robotics and Automation (IEEE) pp 2336-41

[9] Tolley M T, Shepherd R F, Mosadegh B, Galloway K C, Wehner M, Karpelson M, Wood R J and Whitesides G M 2014 A resilient, untethered soft robot Soft Robot. 1 213-23

[10] Albu-Schaffer A, Eiberger O, Grebenstein M, Haddadin S, Ott C, Wimbock T, Wolf S and Hirzinger G 2008 Soft robotics IEEE Robot. Autom. Mag. 15 20-30

[11] Van Ham R, Sugar T G, Vanderborght B, Hollander K W and Lefeber D 2009 Compliant actuator designs IEEE Robot. Autom. Mag. 1681-94

[12] Laschi C and Cianchetti M 2014 Soft robotics: new perspectives for robot bodyware and control Frontiers Bioeng. Biotechnol. 23

[13] Rahman S M 2012 A novel variable impedance compact compliant series elastic actuator for human-friendly soft robotics applications IEEE RO-MAN (IEEE) pp 19-24

[14] Tsagarakis N G, Laffranchi M, Vanderborght B and Caldwell D G 2009 A compact soft actuator unit for small scale human friendly robots IEEE Int. Conf. on Robotics and Automation (IEEE) pp 4356-62

[15] Philamore H, Rossiter J, Stinchcombe A and Ieropoulos I 2015 Row-bot: an energetically autonomous artificial water boatman IEEE/RSJ Int. Conf. on Intelligent Robots and Systems (IEEE) pp 3888-93

[16] Sanan S, Moidel J and Atkeson C G 2011 A continuum approach to safe robots for physical human interaction Int. Symp. on Quality of Life Technology (Citeseer)

[17] Hughes J, Culha U, Giardina F, Guenther F, Rosendo A and Iida F 2016 Soft manipulators and grippers: a review Frontiers Robot. AI 369

[18] Wall V, Deimel R and Brock O 2015 Selective stiffening of soft actuators based on jamming IEEE Int. Conf. on Robotics and Automation (IEEE) pp 252-7

[19] Albu-Schäffer A et al 2011 Anthropomorphic soft roboticsfrom torque control to variable intrinsic compliance Robotics Research pp 185-207

[20] Ranzani T, Gerboni G, Cianchetti M and Menciassi A 2015 A bioinspired soft manipulator for minimally invasive surgery Bioinspir. Biomim. 10035008

[21] Cianchetti M, Ranzani T, Gerboni G, Nanayakkara T, Althoefer K, Dasgupta P and Menciassi A 2014 Soft robotics technologies to location shortcomings in today's minimally invasive surgery: the stiff-flop approach Soft Robot. 1 122-31

[22] Anderson I A, Gisby T A, McKay T G, O’Brien B M and Calius E P 2012 Multi-functional dielectric elastomer artificial muscles for soft and smart machines J. Appl. Phys. 112041101

[23] Walker I D, Dawson D M, Flash T, Grasso F W, Hanlon R T, Hochner B, Kier W M, Pagano C C, Rahn C D and Zhang Q M 2005 Continuum robot arms inspired by cephalopods Unmanned Ground Vehicle Technology VII vol 5804 (International Society for Optics and Photonics) pp 303-14

[24] Gerboni G, Diodato A, Ciuti G, Cianchetti M and Menciassi A 2017 Feedback control of soft robot actuators via commercial flex bend sensors IEEE/ASME Trans. Mechatronics 22 1881-8

[25] Marchese A D, Komorowski K, Onal C D and Rus D 2014 Design and control of a soft and continuously deformable 2D robotic manipulation system IEEE Int. Conf. on Robotics and
Automation (Institute of Electrical and Electronics Engineers (IEEE)) (https://doi.org/10.1109/ICRA.2014.6907161)

[26] Trivedi D, Rahn C D, Kier W M and Walker I D 2008 Soft robotics: Biological inspiration, state of the art, and future research Appl. Bionics Biomech. 599-117

[27] Baumgart F 2000 Stiffness - an unknown world of mechanical science? Injury -Int. J. Care Injured 31 14-23

[28] Howell L L, Magleby S P and Olsen B M 2013 Handbook of Compliant Mechanisms (Hoboken, NJ: Wiley) (https://doi. org/10.1002/9781118516485)

[29] Cheng N G, Gopinath A, Wang L, Iagnemma K and Hosoi A E 2014 Thermally tunable, self-healing composites for soft robotic applications Macromol. Mater. Eng. 299 1279-84

[30] Cheng N, Ishigami G, Hawthorne S, Chen H, Hansen M, Telleria M, Playter R and Iagnemma K 2010 Design and analysis of a soft mobile robot composed of multiple thermally activated joints driven by a single actuator IEEE Int. Conf. on Robotics and Automation (IEEE) pp 5207-12

[31] Kim Y-J, Cheng S, Kim S and Iagnemma K 2014 A stiffnessadjustable hyperredundant manipulator using a variable neutral-line mechanism for minimally invasive surgery IEEE Trans. Robot. $30382-95$

[32] Li S, Vogt D M, Rus D and Wood R J 2017 Fluid-driven origami-inspired artificial muscles Proc. Natl Acad. Sci. 11413132-7

[33] Correll N, Önal Ç D, Liang H, Schoenfeld E and Rus D 2014 Soft autonomous materials - using active elasticity and embedded distributed computation Experimental Robotics (Berlin: Springer) pp 227-40

[34] Rus D and Tolley M T 2018 Design, fabrication and control of origami robots Nat. Rev. Mater. 3101

[35] Fang H, Zhang Y and Wang K 2017 Origami-based earthworm-like locomotion robots Bioinspir. Biomim. 12065003

[36] Rafsanjani A, Zhang Y, Liu B, Rubinstein S M and Bertoldi K 2018 Kirigami skins make a simple soft actuator crawl Sci. Robot. 3 eaar 7555

[37] Mark A G, Palagi S, Qiu T and Fischer P 2016 Auxetic metamaterial simplifies soft robot design IEEE Int. Conf. on Robotics and Automation (IEEE) pp 4951-6

[38] Silverberg J L, Evans A A, McLeod L, Hayward R C, Hull T, Santangelo C D and Cohen I 2014 Using origami design principles to fold reprogrammable mechanical metamaterials Science 345 647-50

[39] Neville R M, Scarpa F and Pirrera A 2016 Shape morphing kirigami mechanical metamaterials Sci. Rep. 631067

[40] Mirzaali M, Janbaz S, Strano M, Vergani L and Zadpoor A A 2018 Shape-matching soft mechanical metamaterials Sci. Rep. 8965

[41] Kim K, Agogino A K, Moon D, Taneja L, Toghyan A, Dehghani B, SunSpiral V and Agogino A M 2014 Rapid prototyping design and control of tensegrity soft robot for locomotion IEEE Int. Conf. on Robotics and Biomimetics (IEEE) pp 7-14

[42] Kimber J, Ji Z, Petridou A, Sipple T, Barhydt K, Boggs J, Dosiek L and Rieffel J 2019 Low-cost wireless modular soft tensegrity robots 2 nd IEEE Int. Conf. on Soft Robotics (RoboSoft) (IEEE) pp 88-93

[43] Mintchev S, Zappetti D, Willemin J and Floreano D 2018 A soft robot for random exploration of terrestrial environments IEEE Int. Conf. on Robotics and Automation (IEEE) pp 7492-7

[44] Vespignani M, Friesen J M, SunSpiral V and Bruce J 2018 Design of superball v2, a compliant tensegrity robot for absorbing large impacts In 2018 IEEE/RSJ Int. Conf. on Intelligent Robots and Systems (IEEE) pp 2865-71

[45] Tondu B and Lopez P 2000 Modeling and control of McKibben artificial muscle robot actuators IEEE Control Systems 20 15-38

[46] Bao G, Fang H, Chen L, Wan Y, Xu F, Yang Q and Zhang L 2018 Soft robotics: academic insights and perspectives through bibliometric analysis Soft Robot. 5 229-41

[47] Wang L and Iida F 2015 Deformation in soft-matter robotics: a categorization and quantitative characterization IEEE Robot. Autom. Mag. 22 125-39 
[48] Wang Let al 2017 Soft-material robotics Found. Trends Robot. 5 191-259

[49] Kastor N, Vikas V, Cohen E and White R D 2017 A definition of soft materials for use in the design of robots Soft Robot. 4 181-2

[50] Sugiyama Y and Hirai S 2004 Crawling and jumping of deformable soft robot IEEE/RSJ Int. Conf. on Intelligent Robots and Systems vol 4 (IEEE) pp 3276-81

[51] Akella P and Cutkosky M 1989 Manipulating with soft fingers: modeling contacts and dynamics IEEE Int. Conf. Robotics and Automation (IEEE) pp 764-9

[52] Iida F and Laschi C 2011 Soft robotics: challenges and perspectives Proc. Comput. Sci. 7 99-102

[53] Hosoda K, Tada Y and Asada M 2006 Anthropomorphic robotic soft fingertip with randomly distributed receptors Robot.Auton. Syst. 54 104-9

[54] Doi M 2013 Soft Matter Physics (Oxford: Oxford University Press) (https://doi.org/10.1093/acprof:o so/9780199652952.001.0001)

[55] McNaught A D 1997 Compendium of Chemical Terminology vol 1669 (Research Triangle Park, NC: Blackwell Science Oxford) (https://doi.org/10.1002/actp.1988.010390322)

[56] Hamley I W 2013 Introduction to Soft Matter and Biological Self-Assembling Materials (New York: Wiley) (https://doi. org/10.1002/9780470517338)

[57] Zhang K, Qiu C and Dai J S 2015 Helical kirigami-enabled centimeter-scale worm robot with shape-memory-alloy linear actuators J. Mech. Robot. 7021014

[58] Wei Y, Chen Y, Ren T, Chen Q, Yan C, Yang Y and Li Y 2016 A novel, variable stiffness robotic gripper based on integrated soft actuating and particle jamming Soft Robot. 3 134-43

[59] Martinez RV, Fish CR, Chen X and Whitesides GM 2012 Elastomeric origami: programmable paper-elastomer composites as pneumatic actuators Adv. Funct. Mater. 22 1376-84

[60] Lazarus A and Reis P M 2015 Soft actuation of structured cylinders through auxetic behavior Adv. Eng. Mater. 17 815-20
[61] Onal C D and Rus D 2013 Autonomous undulatory serpentine locomotion utilizing body dynamics of a fluidic soft robot Bioinspir. Biomim. 8026003

[62] Wehner M, Tolley M T, Mengüç Y, Park Y-L, Mozeika A, Ding Y, Onal C, Shepherd R F, Whitesides G M and Wood R J 2014 Pneumatic energy sources for autonomous and wearable soft robotics Soft Robot. 1 263-74

[63] Preston D J, Rothemund P, Jiang H J, Nemitz M P, Rawson J, Suo Z and Whitesides G M 2019 Digital logic for soft devices Proc. Natl. Acad. Sci. 1167750-9

[64] ISO 83732012 Robots and Robotic Devices-Vocabulary (International Organisation for Standardization)

[65] Rich S I, Wood R J and Majidi C 2018 Untethered soft robotics Nat. Electron. 1102

66] Pfeifer R, Lungarella M and Iida F 2012 The challenges ahead for bio-inspired 'soft' robotics Commun. ACM $5576-87$

[67] Beer J M, Fisk A D and Rogers W A 2014 Toward a framework for levels of robot autonomy in human-robot interaction J. Hum.-Robot Interact. 374-99

[68] Polygerinos P, Correll N, Morin S A, Mosadegh B, Onal C D, Petersen K, Cianchetti M, Tolley M T and Shepherd R F 2017 Soft robotics: Review of fluid-driven intrinsically soft devices; manufacturing, sensing, control, and applications in humanrobot interaction Adv. Eng. Mater. 191700016

[69] Lee C, Kim M, Kim Y J, Hong N, Ryu S, Kim H J and Kim S 2017 Soft robot review Int. J. Control Autom. Syst. 15 3-15

[70] Askeland D R and Wright W J 2013 Essentials of Materials Science (Mason, $\mathrm{OH}$ : Cengage Learning)

[71] McKee C T, Last J A, Russell P and Murphy C J 2011 Indentation versus tensile measurements of Young's modulus for soft biological tissues Tissue Eng. B 17 155-64

[72] Elango N and Faudzi A 2015 A review article: investigations on soft materials for soft robot manipulations Int. J. Adv. Manuf. Technol. 80 1027-37 